\title{
The MiniBooNE anomaly and heavy neutrino decay
}

\author{
S.N. Gninenko \\ Institute for Nuclear Research of the Russian Academy of Sciences, Moscow 117312
}

(Dated: October 23, 2018)

\begin{abstract}
The anomaly in the low energy distribution of quasi-elastic neutrino events reported by the MiniBooNE collaboration is discussed. We show that the observed excess of electron-like events could originate from the production and decay of a heavy neutrino $\left(\nu_{h}\right)$ in the MiniBooNE detector. The $\nu_{h}$ is created by mixing in $\nu_{\mu}$ neutral-current interactions and decays radiatively into $\nu \gamma$ due to a transition magnetic moment between the $\nu_{h}$ and a light neutrino $\nu$. The energy measured in the detector arises from the subsequent conversion of the decay photon into a $e^{+} e^{-}$pair within the detector volume. The analysis of the energy and angular distributions of the excess events suggests that the $\nu_{h}$ has a mass around $500 \mathrm{MeV}$ and the lifetime $\tau_{\nu_{h}} \lesssim 10^{-9} \mathrm{~s}$. Existing experimental data are found to be consistent with a mixing strength between the $\nu_{h}$ and the $\nu_{\mu}$ of $\left|U_{\mu h}\right|^{2} \simeq(1-4) \times 10^{-3}$ and a $\nu_{h}$ transition magnetic moment of $\mu_{t r} \simeq(1-6) \times 10^{-9} \mu_{B}$. Finally, we discuss the reason why no significant excess of low energy events has been observed in the recent antineutrino data.
\end{abstract}

PACS numbers: 14.80.-j, 12.60.-i, 13.20.Cz, 13.35.Hb

The MiniBooNE collaboration, which was searching for the LSND signal from neutrino oscillations at FNAL, has observed an excess of low energy electron-like events in charge-current quasi-elastic $(C C Q E)$ neutrino events over the expected standard neutrino interactions [1]. This anomaly has been recently confirmed by the finding of more excess events [2]. While the collaboration has not yet clarified the origin of the excess, several models involving new physics were considered to explain the discrepancy [3].

In this work we show that the excess could be explained by the production and decay of a heavy neutrino $\left(\nu_{h}\right)$. Such type of neutrinos are present in many interesting extensions of the Standard Model, such as GUT, Superstring inspired models, Left-Right Symmetric models and others. The massive neutrino decays were also considered to explain the LSND signal [4].

The neutrino weak flavor eigenstates $\left(\nu_{e}, \nu_{\mu}, \nu_{\tau}, \ldots\right)$ can be different from the mass eigenstates $\left(\nu_{1}, \nu_{2}, \nu_{3}, \nu_{4} \ldots\right)$, but they are related to them, in general, through a unitary transformation. A generalized mixing:

$$
\nu_{l}=\sum_{i} U_{l i} \nu_{i} ; \quad l=e, \mu, \tau, \ldots, i=1,2,3,4, \ldots
$$

results in neutrino oscillations when the mass differences are small, and in neutrino decays when the mass differences are large. If the $\nu_{h}$ exists, it could be a component of $\nu_{\mu}$, and, as follows from Eq.(1), it would be produced by any source of $\nu_{\mu}$ according to the proper mixing $\left|U_{\mu h}\right|^{2}$ and kinematic constraints. The $\nu_{h}$ can be Dirac or Majorana type [5] and can decay radiatively into $\nu \gamma$, if there is a non-zero transition magnetic moment between the $\nu_{h}$ and a light neutrino $\nu_{i}[\underline{6}$.

The MiniBooNE detector is described in details in Ref. 7]. It is using an almost pure $\nu_{\mu}$ beam originated from the $\pi^{+}$decays in flight, which are generated by $8 \mathrm{GeV}$ protons from the FNAL booster. The detector consists of a target, which is a $12.2 \mathrm{~m}$ diameter sphere filled with
$800 \mathrm{t}$ of mineral oil, surrounded by an outer veto region. The Cherenkov light rings generated by muon, electron and converted photon tracks are used for the reconstruction of the events. The resolutions reached on the vertex position, the outgoing particle direction and the visible energy are $20 \mathrm{~cm}, 4$ degrees and $12 \%$, respectively for $C C Q E$ electrons [8]. The $\nu_{\mu}$ beam is peaked around $\sim 600 \mathrm{MeV}$, has a mean energy of $\sim 800 \mathrm{MeV}$ and a high energy tail up to $\sim 3 \mathrm{GeV}[9]$.

An excess of $\Delta N=128.8 \pm 20.4 \pm 38.3$ electron-like events has been observed in the data accumulated with $6.64 \times 10^{20}$ protons on target. For the following discussion several distinctive features of the excess events are of importance [2]: a) the excess is observed for single track events, originating either from an electron, or from a photon converted into a $e^{+} e^{-}$pair with a typical opening angle $\simeq m_{e} / E_{e^{+} e^{-}}<1$ degree (for $E_{e^{+} e^{-}}>200$ $\mathrm{MeV}$ ), which is too small to be resolved into two separate Cherenkov rings (here, $m_{e}, E_{e^{+} e^{-}}$are the electron mass and the $e^{+} e^{-}$pair energy); b) the reconstructed neutrino energy is in the range $200<E_{\nu}^{Q E}<475 \mathrm{MeV}$, while there is no significant excess for the region $E_{\nu}^{Q E}>475$ $\mathrm{MeV}$. The variable $E_{\nu}^{Q E}$ is calculated under the assumption that the observed electron track originates from a $\nu_{e} Q E$ interaction; c) the visible energy $E_{v i s}$ is in the narrow region $200 \lesssim E_{v i s} \lesssim 400 \mathrm{MeV}$ for events with $E_{\nu}^{Q E}>200 \mathrm{MeV}$; d) the angular distribution of the excess events with respect to the incident neutrino direction is wide and consistent with the shape expected from $\nu_{e} C C$ interactions. To satisfy the criteria a)-d), we propose that the excess events are originated from the decay of a heavy neutrino $\nu_{h}$. The $\nu_{h}$ 's are produced by mixing in $\nu_{\mu}$ neutral-current $(N C) Q E$ interactions and deposit their energy via the visible decay mode $\nu_{h} \rightarrow \nu \gamma$, as shown in Fig.1, with the subsequent conversion of the decay photon into $e^{+} e^{-}$pair in the MiniBooNE target. To make a quantitative estimate, we performed simplified simulations of the production and decay processes shown in Fig.1. In these simulations we used a $\nu_{\mu}$ energy 


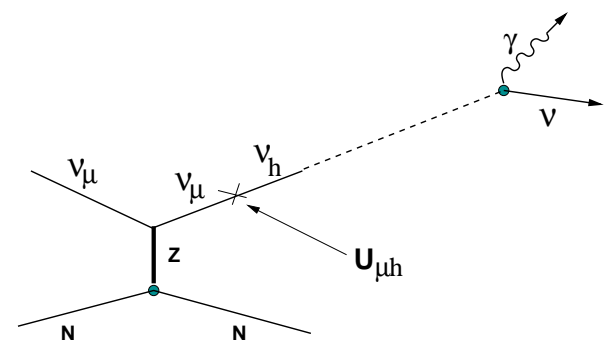

FIG. 1: Schematic illustration of the NCQE production and decay of heavy neutrino.

spectrum parametrized from the reconstructed $\nu_{\mu} C C Q E$ events [9]. Since in the MiniBooNE experiment $\nu_{h}$ 's decay over an average distance of $\lesssim 5 \mathrm{~m}$ from the production vertex, the sensitivity is restricted to the mass range $100-600 \mathrm{MeV}$ and to $\nu_{h}$ lifetimes $\tau_{\nu_{h}}<10^{-7} \mathrm{~s}$.

In Fig 24 the distributions of the kinematic variables $E_{\nu}^{Q E}, E_{v i s}$ and $\cos \left(\Theta_{\gamma \nu}\right)$ for the $\nu_{h} \rightarrow \gamma \nu$ events are shown for $m_{\nu_{h}}=400$ and $600 \mathrm{MeV}$ and $\tau_{\nu_{h}}=3 \times 10^{-8}$ and $10^{-10} \mathrm{~s}$. These distributions were obtained assuming that the $e^{+} e^{-}$pair from the converted photon is misreconstructed as a single track from the $\nu_{e} Q E$ reaction.

Simulations are in reasonable agreement with the experimental distributions. For instance, for the distribution shown in Fig 2 the comparison with MiniBooNE data yields a $\chi^{2}$ of $10.2(17.2)$ for $8 \mathrm{DF}$ corresponding to $27 \%(\simeq 5 \%)$ CL. for $m_{\nu_{h}}=400(600) \mathrm{MeV}$ and $\tau_{\nu_{h}}=3 \times 10^{-8} \mathrm{~s}$. The simulated excess events, shown in Fig 3 are mainly distributed in the narrow region $200 \lesssim E_{v i s} \lesssim 400 \mathrm{MeV}$. The fraction of events in the region $200<E_{\text {vis }}<400 \mathrm{MeV}$ is $\sim 70 \%$. The remaining events are distributed over the region $400 \lesssim E_{\text {vis }} \lesssim 1200$ $\mathrm{MeV}$, where they can be hidden by the low statistics. The simulations showed that the shape of the $E_{\nu}^{Q E}$ and $E_{v i s}$ distributions is sensitive to the choice of the $\nu_{h}$ mass and lifetime: the shorter the $\nu_{h}$ lifetime the broader the visible energy spectrum. The best fit results suggest that the $\nu_{h}$ mass is in the region $200 \lesssim m_{\nu_{h}} \lesssim 600 \mathrm{MeV}$ and the lifetime is $\tau_{\nu_{h}}<10^{-7} \mathrm{~s}$. The estimate of the mixing parameter $\left|U_{\mu h}\right|^{2}$ was performed by using the following relations. For a given flux of heavy neutrinos, $\Phi\left(\nu_{h}\right)$, the expected number of the decays in the MiniBooNE detector is given by $\Delta N=\int \Phi\left(\nu_{h}\right) P_{d e c} P_{\text {conv }} \epsilon d E_{\nu_{h}} d V$, where $P_{d e c}$ and $P_{\text {conv }}$ are the probabilities of the $\nu_{h}$ decay and the photon conversion in the detector, $\epsilon$ is the overall detection efficiency, and the integral is taken over the detector fiducial volume.

The flux $\Phi\left(\nu_{h}\right)$ was estimated from the expected number of the $\nu_{\mu} N C$ events times the mixing $\left|U_{\mu h}\right|^{2}$, taking into account the threshold effect due to the heavy neutrino mass. The total number of reconstructed $\nu_{\mu} C C$ events in the detector [9] was used for normalization. The probability of the heavy neutrino to decay radiatively in the fiducial volume at a distance $r$ from the primary vertex is given by $P_{d e c}=\left[1-\exp \left(\frac{-r m_{\nu_{h}}}{p_{\nu_{h}} \tau_{\nu_{h}}}\right)\right] \frac{\Gamma\left(\nu_{h} \rightarrow \gamma \nu\right)}{\Gamma_{\text {tot }}}$,

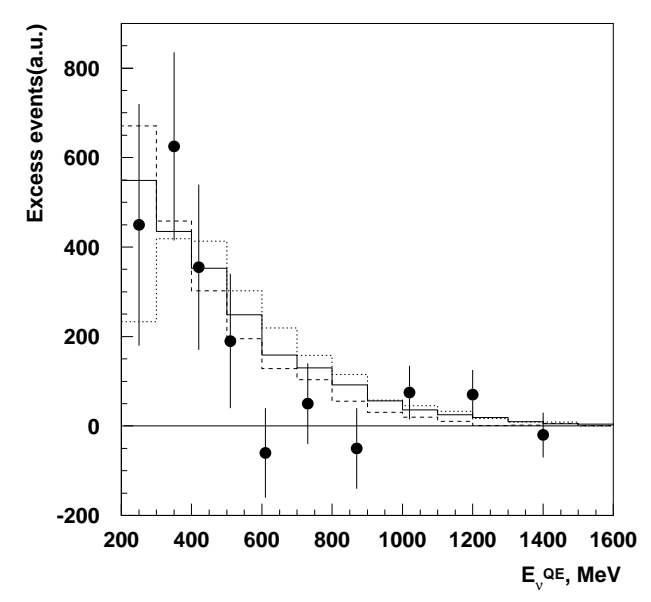

FIG. 2: Distributions of the excess events from the $\nu_{h} \rightarrow \gamma \nu$ decay reconstructed as $\nu_{e} C C$ events as a function of $E_{\nu}^{Q E}$ for $\left|U_{\mu h}\right|^{2}=1.5 \times 10^{-3}$ and a) $m_{\nu_{h}}=400$ and $\tau_{\nu_{h}}=3 \times 10^{-8} \mathrm{~s}$ $\left(\mu_{t r}=2 \times 10^{-10} \mu_{B}\right)($ solid $) ;$ b) $m_{\nu_{h}}=400$ and $\tau_{\nu_{h}}=10^{-10} \mathrm{~s}$ $\left(\mu_{t r}=3 \times 10^{-9} \mu_{B}\right)($ dashed $)$; c) $m_{\nu_{h}}=600$ and $\tau_{\nu_{h}}=3 \times 10^{-8}$ $\mathrm{s}$ (dotted). The dots are experimental points for the excess events in the MiniBooNE detector. Error bars include both statistical and systematic errors [2]. The comparison of the distributions with the experimental data yields a $\chi^{2}$ of 10.2 , 11.2 , and 17.2 for $8 \mathrm{DF}$ corresponding to $27 \%, 24 \%$, and $\simeq$ $5 \%$ C.L. for a),b) and c), respectively.

where the last term is the branching fraction $\operatorname{Br}\left(\nu_{h} \rightarrow\right.$ $\gamma \nu) \simeq 1$ (see below). Taking into account the ratio $\nu_{\mu} N C Q E / \nu_{\mu} C C Q E \sim 0.43$, the number of $\nu_{\mu} C C Q E$ events observed [1, 2] and assuming that almost all $\nu_{h} \rightarrow \gamma \nu$ decays occur inside the fiducial volume of the detector, we estimate the $\left|U_{\mu h}\right|^{2}$ to be in the range

$$
\left|U_{\mu h}\right|^{2} \simeq(1-4) \times 10^{-3} .
$$

This result is mainly defined by the uncertainty on the number of excess events. Eq.(21) is valid for the mass region $400 \lesssim m_{\nu_{h}} \lesssim 600 \mathrm{MeV}$. The lower limit is set to $400 \mathrm{MeV}$ to avoid stringent constraints on $\left|U_{\mu h}\right|^{2}$ for the mass region $m_{\nu_{h}} \lesssim 400 \mathrm{MeV}$ from experiments searching for a peak from $\pi, K \rightarrow \mu+\nu_{h}$ decays [10]. The $\nu_{h}$ lifetime due to a transition moment $\mu_{t r}$ is given by [6]

$$
\tau_{\nu \gamma}^{-1}=\frac{\alpha}{8}\left(\frac{\mu_{t r}}{\mu_{B}}\right)^{2}\left(\frac{m_{\nu_{h}}}{m_{e}}\right)^{2} m_{\nu_{h}}
$$

and for $\tau_{\gamma \nu}<10^{-7} \mathrm{~s}$ results in $\mu_{t r}>10^{-10} \mu_{B}$. The total $\nu_{h}$ decay width is $\Gamma_{t o t}=\Gamma\left(\nu_{h} \rightarrow \nu \gamma\right)+\Sigma \Gamma_{i}$, where $\Gamma\left(\nu_{h} \rightarrow \nu \gamma\right)$ is the $\nu_{h} \rightarrow \gamma \nu$ decay rate, and $\Sigma \Gamma_{i}$ is the sum over decay modes whose decay rate is proportional to the square of the mixing $\left|U_{\mu h}\right|^{2}$. The dominant contribution to $\Sigma \Gamma_{i}$ comes from $\nu_{h} \rightarrow \nu_{\mu} e e, \nu_{\mu} \pi^{0}, \nu_{\mu} \nu \nu, \mu \pi$ decays, for which the rate calculations can be found, e.g. in [11]. For $m_{\nu_{h}} \simeq 500 \mathrm{MeV}$ and $\mu_{t r}>10^{-10} \mu_{B}$, we found that the radiative decay is dominant, $\operatorname{Br}\left(\nu_{h} \rightarrow\right.$ $\gamma \nu)>0.5$. For example, for $\mu_{t r}=10^{-9} \mu_{B}$ and 


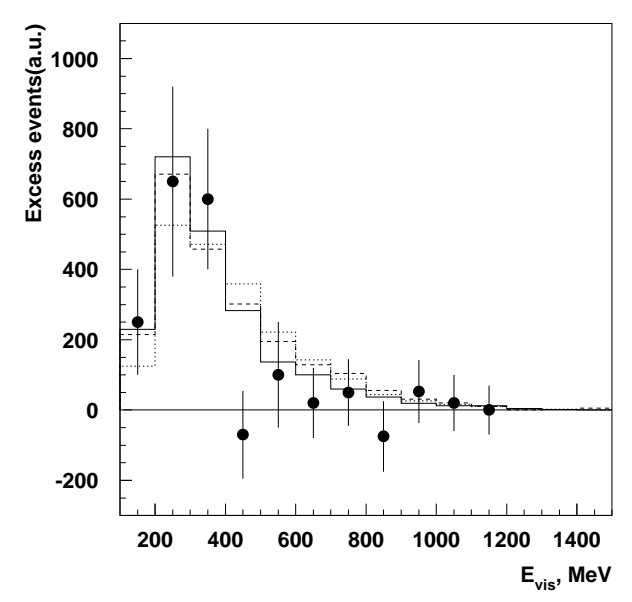

FIG. 3: Distributions of the excess events from the $\nu_{h} \rightarrow \gamma \nu$ decay reconstructed as $\nu_{e} C C$ events as a function of $E_{v i s}$ for $E_{\nu}^{Q E}>200 \mathrm{MeV}$. The comparison of the distributions with the experimental data yield a $\chi^{2}$ of $9.7,10.3$, and 16.8 for 8 DF corresponding to $28 \%, 27 \%$, and $\simeq 5 \%$ C.L. for a), b) and c), respectively. The legend is the same as in Fig 2 .

$\left|U_{\mu h}\right|^{2}=1.5 \times 10^{-3}$, the expected ratio of decay rates for $\gamma \nu: \mu \pi$ : e $е \nu \nu$ : $\mu \mu \nu$ is $0.984: 0.011: 0.0016$ : 0.00067 .

One may wonder if the mixing strength of Eq.(2) is consistent with the results of previous searches for $\nu_{h}$ decays. The $\nu_{h}$ mass region around $500 \mathrm{MeV}$ was covered by many experiments [10, 11, 12]. However, none of these experiments has reported a bound on the mixing strength $\left|U_{\mu h}\right|^{2}$ or on the combination $\left|U_{\mu h}\right|^{2} \mu_{t r}$, for the radiative $\nu_{h} \rightarrow \gamma \nu$ neutrino decay. The best limit $\left|U_{\mu h}\right|^{2} \lesssim 10^{-6}$ for the mass region $m_{\nu_{h}} \simeq 500 \mathrm{MeV}$ was derived from a search for $\nu_{h} \rightarrow \mu \pi, \mu \mu \nu, \mu e \nu$ decays in the $\mathrm{NuTeV}$ beam dump experiment 13] (see also [14, 15, 16]). It was assumed that these decay modes are dominant and that the $\nu_{h}$ is a relatively long lived particle, i.e $\frac{L m_{\nu_{h}}}{p_{\nu_{h}} \tau_{\nu_{h}}} \ll 1$, where $L \simeq 1.4 \times 10^{3} \mathrm{~m}$ is the distance between the target and the detector. Consider now our case with $\left|U_{\mu h}\right|^{2}=(1-4) \times 10^{-3}, m_{\nu_{h}}=500$ $\mathrm{MeV}$ and $\mu_{t r}=10^{-9} \mu_{B}$. This gives the $\nu_{h}$ lifetime $\tau_{\nu_{h}}=(1.5-1.4) \times 10^{-9} \mathrm{~s}$. Due to the larger mixing the $\nu_{h}$ flux at the target would increase by a factor $\simeq(1-4) \times 10^{3}$. However, taking into account the attenuation of the flux due to the rapid decay of $\nu_{h}$ 's, the total number of signal events in $\mathrm{NuTeV}$ would decrease by a factor (10-3) compared to the number of events expected for a long lived $\nu_{h}$ 's produced and decaying through the mixing $\left|U_{\mu h}\right|^{2}=10^{-6}$. In this estimate the average $\nu_{h}$ momentum is $\left\langle p_{\nu_{h}}>\simeq 100 \mathrm{GeV}\right.$ and the decay region length is $l=34 \mathrm{~m} \mathrm{[13]}$. Finally, we find that for

$$
\mu_{t r} \gtrsim 10^{-9} \mu_{B}
$$

the NuTeV limit is not constraining mixing of Eq.(2). Note that a short $\nu_{h}$ lifetime is also necessary to avoid the

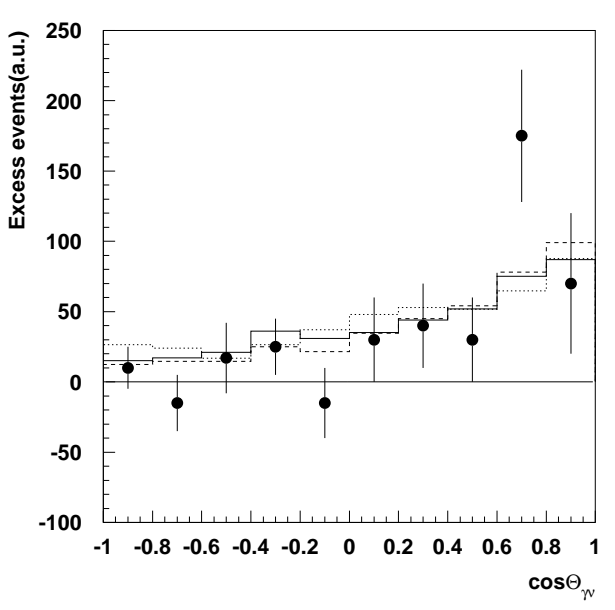

FIG. 4: Distribution of the excess events from the $\nu_{h}$ decay reconstructed as $\nu_{e} C C$ events as a function of $\cos \Theta_{\gamma \nu}$ for $300<E_{\nu}^{Q E}<400 \mathrm{MeV}$. The comparison of the distributions with the experimental data yields a $\chi^{2}$ of 11.6, 11.1, and 15.6 for 8 DF corresponding to $23 \%, 24 \%$, and $\simeq 5 \%$ C.L. for a), b) and c), respectively. The notations are the same as in Fig 2 .

constraints coming from cosmological and astrophysical considerations [17].

The best limit for the large mixing (short lifetimes), $\left|U_{\mu h}\right|^{2} \lesssim 10^{-3}$ for the masses around $500 \mathrm{MeV}$, was derived by CHARM-II from a search for the $\nu_{h}$ production and $\nu_{h} \rightarrow \mu \mu \nu$ decays within their detector [15]. Taking into account the $\nu_{h} \rightarrow \gamma \nu$ decay, the $\left\langle p_{\nu_{h}}\right\rangle \simeq 24$ $\mathrm{GeV}$ and $l=35 \mathrm{~m}$, we find that the number of the expected $\mu \mu \nu$ signal events in CHARM-II is $N_{\mu \mu \nu} \simeq$ $0.46 \times 10^{-12} \frac{\left|U_{\mu h}\right|^{4}}{\left(\mu_{t r} / \mu_{B}\right)^{2}}$. For $\mu_{t r} \geq 3 \times 10^{-9} \mu_{B}$ and for mixing of Eq.(2) this results in $N_{\mu \mu \nu}<0.05-0.8$ events. To evade the CHARM-II limit for the region $10^{-9}<\mu_{t r}<$ $3 \times 10^{-9} \mu_{B}$ the mixing is required to be in a slightly more restricted range $10^{-3}<\left|U_{\mu h}\right|^{2}<1.5 \times 10^{6} \frac{\mu_{t r}}{\mu_{B}}$ compare to that of Eq.(2). For example, for $\mu_{t r}=2 \times 10^{-9} \mu_{B}$ the allowed range is $10^{-3}<\left|U_{\mu h}\right|^{2}<3 \times 10^{-3}$. Thus, we see that most of the allowed $\left(\mu_{t r} ;\left|U_{\mu h}\right|^{2}\right)$ parameter space corresponding to Eqs.(24) is not constrained by the CHARM-II limit.

Consider now bounds from LEP experiments [10]. For the mass region around $500 \mathrm{MeV}$, the model independent limit from the searches for the $Z \rightarrow \nu \nu_{h}$ decay is $\left|U_{\mu h}\right|^{2} \lesssim$ $10^{-2}$, (see e.g. [18]) which is compatible with Eq.(2). Direct searches for radiative decays of an excited neutrino $\nu^{*} \rightarrow \gamma \nu$ produced in $Z \rightarrow \nu^{*} \nu$ decays have been also performed [10]. The best limit from ALEPH is $\operatorname{Br}(Z \rightarrow$ $\left.\nu \nu^{*}\right) \operatorname{Br}(\nu * \rightarrow \gamma \nu)<2.7 \times 10^{-5}[19]$. As the experimental signatures for the $\nu * \rightarrow \gamma \nu$ and $\nu_{h} \rightarrow \gamma \nu$ decays are the same, we will use this bound for comparison. The number of expected $\nu_{h} \rightarrow \gamma \nu$ events in ALEPH is proportional to $\operatorname{Br}\left(Z \rightarrow \nu \nu_{h}\right) \operatorname{Br}\left(\nu_{h} \rightarrow \gamma \nu\right)\left[1-\exp \left(-\frac{l m_{\nu_{h}}}{p_{\nu_{h}} \tau_{\nu_{h}}}\right)\right]$, with $l \simeq 1$ 
$\mathrm{m}$ and $p_{\nu_{h}} \simeq 45 \mathrm{GeV}$. Taking into account $\frac{B r\left(Z \rightarrow \nu \nu_{h}\right)}{B r(Z \rightarrow \nu \nu)} \simeq$ $\left|U_{\mu h}\right|^{2}$ and using Eq.(3), we find

$$
\left|U_{\mu h}\right|^{2} \times\left(\frac{\mu_{t r}}{\mu_{B}}\right)^{2}<3.5 \times 10^{-20} .
$$

Using Eq. (2) results in $\mu_{t r} \lesssim(6-3) \times 10^{-9} \mu_{B}$, which is consistent with Eq.(41).

The limit on the $\mu_{t r}$ between the $\nu_{h}$ and the $\nu_{\mu}$ has been obtained in Ref.[20], based on the idea of the Primakoff conversion $\nu_{\mu} Z \rightarrow \nu_{h} Z$ of the muon neutrino into a heavy neutrino in the external Coulomb field of a nucleus $Z$, with the subsequent $\nu_{h} \rightarrow \gamma \nu$ decay. By using the results from the NOMAD experiment [21, 22], a model-independent bound $\mu_{t r}^{\mu h} \lesssim 10^{-8} \mu_{B}$ was set for the $\nu_{h}$ masses around $500 \mathrm{MeV}$ (see Table 1 and Fig. 2 in Ref.[20]), which is also consistent with Eq.(4).

The low statistics anti-neutrino $\left(\bar{\nu}_{\mu}\right)$ data collected by the MiniBooNe seem to show no low-energy excess [23]. An analysis of these data within the framework discussed above suggests that the excess is not seen due to the lower $\bar{\nu}_{\mu}$ energy. Indeed, the $\bar{\nu}_{\mu}$ flux peaks at $\sim 400 \mathrm{MeV}$ and has a mean energy of $\sim 600 \mathrm{MeV}$ [9]. If the $\bar{\nu}_{h}$ mass is around $500 \mathrm{MeV}$, the $\bar{\nu}_{h}$ production is kinematically suppressed for $\bar{\nu}_{\mu}$ energies below the mean energy. Instead of the expected excess of $\sim 40$ events [23], a smaller excess of $\sim 23$ events is expected in the antineutrino data.

In summary, we see that the interpretation of the Mini-
BooNe anomaly based on the production and visible decay of a heavy neutrino is compatible with all the four constraints a)-d). The shape of the excess events in several kinematic variables is found to be consistent with the distributions obtained within this interpretation. The reason why the excess is not observed in the recent antineutrino data [23] is clarified. A definite conclusion on the presence of $\bar{\nu}_{h} \rightarrow \bar{\nu}_{\mu} \gamma$ events can be drawn when the $\bar{\nu}_{\mu}$ statistics is substantially increased. Our results for the mixing strength $\left|U_{\mu h}\right|^{2} \simeq(1-4) \times 10^{-3}$ and for the magnetic moment $\mu_{t r} \simeq(1-6) \times 10^{-9} \mu_{B}$ are compatible with the results from previous experiments. Values of $\mu_{t r}$ larger than $10^{-10} \mu_{B}$ could be obtained e.g. in the framework of the Zee model [6]. Our analysis gives a correct order of magnitude for the parameters $\left|U_{\mu h}\right|^{2}$ and $\mu_{t r}$ and may be improved by more accurate and detailed simulations of the MiniBooNE detector, which are beyond the scope of this work. We note that an analysis of the excess of events due to the $\nu_{h} \rightarrow \gamma \nu$ decay may also be possible with existing neutrino data. New results could be obtained with NOMAD [21], SciBooNE [24] and K2K near detectors [25], see also 26]. The author thanks S. Brice, A.D. Dolgov, S.H. Hansen, D.S. Gorbunov, N.V. Krasnikov, V.A. Matveev, V.A. Rubakov, M.E. Shaposhnikov, and R. Van de Water for useful discussions and/or comments, and M. Kirsanov, R. Petti and D. Sillou for help. This work was supported by Grant RFBR 08-0291007-CERN.
[1] A.A. Aguilar-Arevalo et al.,Phys. Rev. Lett. 98, 231801 (2007).

[2] A.A. Aguilar-Arevalo et al., arXive:0812.2243.

[3] J. A. Harvey, Ch. T. Hill, and R. J. Hill, Phys. Rev. Lett. 99, 261601 (2007); Phys. Rev. D 77, 085017 (2008); M. Sorel, J. Conrad, and M. Shaevitz, Phys. Rev. D 70, 073004 (2004); C. Karagiori et al. Phys. Rev. D 75, 013011 (2007); A. Melchiorri et al. arXive:0810.5133; M. Maltoni and Th. Schwetz, Phys. Rev. D 76, 093005 (2007). Xue-Qian Li et al., Eur. Phys. J. C 56. 97 (2008); A.E. Nelson and J. Walsh, Phys. Rev. D 77, 033001 (2008); V. Barger, D. Marfatia and K. Whisnant, Phys. Lett. B 576, 303 (2003); T. Katori, A. Kostelesky and R. Tayloe, Phys. Rev. D 74, 1053009 (2006); C. Giunti and M. Laveder, arXiv:0902.1992.

[4] E. Ma, G. Rajasekaran and I.Stancu, Phys. Rev. D 61 , 071302 (2000); E. Ma and G. Rajasekaran, Phys. Rev. D 64, 117303 (2001); S. Palomares-Ruiz, S.Pascoli and Th. Schwetz, JHEP 0509, 048 (2005).

[5] For definitness we will consider further the first case.

[6] See for example, R.N. Mohapatra and P.B. Pal, "Massive Neutrinos in Physics and Astrophysics", World Scientific, Singapore, 1991;

[7] A.A. Aguilar-Arevalo et al., arXive:0806.4201.

[8] R.B. Patterson et al., arXive:0902.2222.
[9] A.A. Aguilar-Arevalo et al., arXive:0806.1449.

[10] C. Amsler et al., Review of Particle Physics, Phys. Lett. B 667, 1 (2008).

[11] L.M. Johnson, D.W. McKay and T. Bolton, Phys. Rev. D 56, 2970 (1997); D. Gorbunov and M. Shaposhnikov, JHEP 0710, 015 (2007).

[12] A. Atre et al., arXive:0901.3589.

[13] A. Vaitaitis et al., Phys. Rev. Lett. 83, 4943 (1999).

[14] A.M. Cooper-Sarkar et al., Phys. Lett. 160B, 207 (1985).

[15] P. Vilain et al., Phys. Lett. 351, 387 (1995).

[16] S. Mishra et al., Phys. Rev. Lett. 59, 1397 (1987).

[17] A.D. Dolgov, Phys. Rept. 370, 333 (2002).

[18] P. Abreu et al., Z. Phys. C 74, 57 (1997).

[19] D. Buskulic et al., Phys. Rep. 216, 253 (1992).

[20] S.N. Gninenko and N.V. Krasnikov, Phys. Lett. B 450, 165 (1999).

[21] J. Altegoer et al., Nucl. Instrum. Meth. A404, 96 (1998).

[22] J. Altegoer et al., Phys. Lett. B 428, 197 (1998); S.N. Gninenko and N.V. Krasnikov, Phys. Lett. B 427, 307 (1998).

[23] A.A. Aguilar-Arevalo et al., arXive:0904.1958.

[24] K. Hiraide et al., Phys. Rev. D 78, 112004 (2008).

[25] T. Ishii et al., Nucl. Instrum. Meth. A 482, 244 (2002).

[26] S.N. Gninenko and D.S. Gorbunov, arXive:0907.4666. 